\title{
Capacidade funcional e suporte familiar em idosos longevos residentes em domicílio
}

\section{Functional capacity and family support in long age elderly home residents}

Como citar este artigo: BÔAS, SARA S. V.; ARAUUJO,

CLAUDINÉIA M.; PRATES, RAHAB $V_{\text {.; }}$ NOVAIS, MARTA M.; PINTO,

DANIELA S.; REIS, LUCIANA A. Capacidade funcional e suporte familiar em idosos longevos residentes em domicilio. Revista Saúde (Sta. Maria). 2020; 46 (1).

\section{Autor correspondente:} Nome: Sara Souza Vilas Bôas E-mail: sariinha_souza@hotmail. com

Telefone: (73)99157-904। Formação Profissional: Formada em Fisioterapia pela Universidade Estadual do Sudoeste da Bahia (UESB), Jequié, BA, Brasil

Filiação Institucional: Universidade Estadual do Sudoeste da Bahia Endereço para correspondencia:

Rua: Antônio Melo Lobo

Bairro: Almir Pereira

Cidade: Gandu

Estado: Bahia

CEP: 45450-000

Data de Submissão:

20/08/20 18

Data de aceite:

01/04/2020

Conflito de Interesse: Não há conflito de interesse

\section{(cc) $\mathrm{BY}-\mathrm{NC}-\mathrm{ND}$}

\author{
Sara Souza Vilas Bôas, Claudinéia Matos de Araújo, Rahab Vieira Prates, \\ Marta Moreira Novais, Daniela de Souza Pinto, Luciana Araújo dos Reis
}

\section{RESUMO}

Objetivo: avaliar a capacidade funcional e o suporte familiar de idosos longevos residentes em domicílio. Métodos: Trata-se de uma pesquisa do tipo descritiva, com delineamento transversal e observacional realizada no município de Jequié com 117 idosos longevos cadastrados em cinco unidades de saúde da família. $O$ instrumento de coleta de dados foi constituído de caracterização sociodemográfica, condições de saúde, Índice de Barthel, Escala de Lawton e Inventário de Percepção de Suporte Familiar, sendo realizada análise descritiva das variáveis de estudo. Resultado: Constatou-se uma maior frequência de idosos do sexo feminino (59,0\%), faixa etária de 80 a 84 anos $(57,3 \%)$ não alfabetizados $(51,3 \%)$, independentes nas atividades básicas de vida diária $(59,0 \%)$, dependentes nas atividades instrumentais de vida diária $(80,3 \%)$, com pontuação alta $(62,4 \%)$ no domínio Afetividade-Consistência, pontuação alta $(53,0 \%)$ no domínio Adaptação Familiar e pontuação alta $(60,7 \%)$ no domínio Autonomia. Constatou-se em relação a capacidade funcional que houve uma predominância de independência para realização das ABVD, enquanto para realização das AIVD foi observada uma dependência. Conclusão: Quanto ao suporte familiar ficou evidenciado que os idosos do estudo possuem comprometimento nos domínios Afetividade-Consistência e Autonomia. Na avaliação das ABVD, verificou- se que a maioria dos idosos foram classificados como independentes e como dependentes nas AIVD.

PALAVRAS-CHAVE: Idoso; Família; Envelhecimento; Funcionalidade.

\section{ABSTRACT}

Objective: to evaluate the functional capacity and family support of long-lived elderly people living at home. Methods: This is a descriptive research, with a cross-sectional and observational design carried out in the municipality of Jequié with 117 long-lived elderly people registered in five family health units. The data collection instrument consisted of sociodemographic characterization, health conditions, Barthel Index, Lawton Scale and Perception Inventory of Family Support, with a descriptive analysis of the study variables. Result: There was a higher frequency of elderly females (59.0\%), aged between 80 and 84 years $(57.3 \%$ ) illiterate $(51.3 \%)$, independent in basic activities of daily living ( $59.0 \%)$, dependent on instrumental activities of daily living (80.3\%), with high score (62.4\%) in the Affectivity-Consistency domain, high score (53.0\%) in the Family Adaptation domain and high score (60.7\%) in the Autonomy domain. It was found in relation to functional capacity that there was a predominance of independence to perform BADLs, while for performing IADLs a dependency was observed. Conclusion: Regarding family support, it was evidenced that the elderly in the study have impairment in the domains Affectivity-Consistency and Autonomy. In the assessment of ABVD, it was found that the majority of the elderly were classified as independent and as dependents in IADL.

KEYWORDS: Elderly; Family; Aging; Functionality. 


\section{INTRODUÇÃO}

O envelhecimento populacional é um fenômeno universal, irreversível e natural, que se relaciona a uma diminuição dos níveis de fecundidade e mortalidade, e a um aumento da população idosa, ou seja, indivíduos com idade igual ou superior a 60 anos ${ }^{1}$. Dentro do grupo das pessoas idosas, a faixa etária que mais cresce no mundo é a de indivíduos com 80 anos ou anos. Esse segmento populacional pode ser denominado como idosos mais idosos, muito idosos, idosos mais velhos, idosos longevos ou octogenários².

Neste contexto, o aumento da sobrevida ocasiona maior prevalência de doenças crônicas, perdas da independência funcional e autonomia. A capacidade funcional pode ser entendida como a habilidade física e mental para manter uma vida independente e autônoma, enquanto, inversamente, a incapacidade funcional refere-se a presença de dificuldade ou até mesmo da impossibilidade no desempenho de determinadas atividades da vida diária (AVD) ${ }^{3}$.

A capacidade funcional está relacionada com a manutenção das atividades básicas de vida diária (ABVD) e atividades instrumentais de vida diária (AIVD), ou seja, com a habilidade de realização de atividades tais como: banhar-se, vestir-se, realizar higiene pessoal, transferir-se, alimentação, manter a continência, preparar refeições, controle financeiro, tomar remédios, arrumar a casa, realizar compras, usar transporte coletivo, usar telefone e caminhar certa distância ${ }^{4}$. A perda da capacidade funcional está associada à predisposição de fragilidade, dependência, institucionalização, risco aumentado de quedas, morte e problemas de mobilidade, acarretando complicações ao longo do tempo e originando cuidados de longa permanência e alto custo 5 .

A cada ano, cerca de $10 \%$ da população adulta, a partir dos 75 anos, perde a independência em uma ou mais $A B V D$, o que, na prática impossibilita que este indivíduo resida ou permaneça em sua residência sozinho. Nesse contexto surge o suporte familiar, que contribui de maneira significativa para a manutenção e a integridade física e psicológica do indivíduo. Sendo assim, é de suma importância para o planejamento assistencial apropriado ao idoso, compreender o seu contexto familiar, o que implica na compreensão das questões que envolvem a formação e a dinâmica de funcionamento das famílias em geral ${ }^{6}$.

O suporte familiar pode ser entendido como o primeiro recurso de socialização por meio do qual os padrões de comportamento, hábitos de linguagem, valores, crenças e costumes são transmitidos e as necessidades psicológicas e fisiológicas básicas podem ser supridas. Um suporte familiar apropriado tem como resultados efeitos emocionais positivos e em sensações de pertencimento, cuidado e estima. Trata-se de um processo mútuo que ocorre entre os membros da família, que promove efeitos tanto para quem recebe, como para quem oferece 0 apoio ${ }^{7}$. A percepção de suporte familiar está relacionada à competência social, capacidade de enfrentamento de problemas, percepção de controle, senso de estabilidade, autoconceito, afeto e, consequentemente, ao bem-estar psicológico8.

Quando a família oferece aos seus integrantes cuidado, carinho, atenção, diálogo, autonomia, empatia, afetividade, aceitação e liberdade, ela funciona como uma importante fonte suporte, imprescindível ao desenvolvimento saudável de seus integrantes ${ }^{9}$. Nessa perspectiva, este estudo teve por objetivo avaliar a capacidade funcional e 0 suporte familiar de idosos longevos residentes em domicílio. 
A população de idosos longevos vem crescendo de forma rápida no país, entretanto a produção científica nessa área não possui o mesmo ritmo. A relevância do presente estudo se dá pelo fato identificar a relação entre suporte familiar e capacidade funcional é essencial para a construção de medidas que visem uma melhor qualidade de vida para esta população.

\section{MÉTODO}

Trata-se de uma pesquisa do tipo descritivo com delineamento transversal, observacional e abordagem quantitativa, realizada no município de Jequié, localizado no interior da Bahia, Região Nordeste do Brasil, no período de junho de 2014 a dezembro de 2014. A amostra foi composta por 117 idosos longevos (80 anos ou mais) cadastrados em cinco unidades de saúde da família (USF) no município de Jequié, sendo que a pesquisa ocorreu a nível domiciliar.

Os critérios de inclusão foram: ser residente em domicílio, possuir capacidade cognitiva de falar e ouvir e ter cognitivo preservado avaliado pelo Mini Exame do Estado Mental (MEEM) com pontuação acima de 24 pontos $^{10}$. Foram considerados como critérios de exclusão a não localização após duas tentativas pelo entrevistador e todos os critérios que não se enquadrar nos itens propostos para inclusão ao estudo. Foram excluídos 19 idosos que apresentaram alterações mentais que impossibilitassem a aplicação do questionário, ou que não foram encontrados no domicílio em três visitas.

O instrumento de coleta de dados foi constituído de: Caracterização sociodemográfica (idade, sexo, escolaridade, profissão, valor da renda e estado civil); Condições de saúde (presença ou ausência de problemas de saúde e de sequelas); Índice de Barthel; Escala de Lawton e Inventário de Percepção de Suporte Familiar.

O índice de Barthel é um instrumento utilizado para avaliar o nível de independência do indivíduo na prática de 10 ABVD: comer, deambular, tomar banho, vestir e despir, higiene pessoal, uso dos sanitários, controle de esfíncteres, transferência da cadeira para a cama, subir e descer escadas, sendo que a quantificação dos pontos na escala varia de 0-100 (com intervalos de 5 pontos). Interpretando os resultados, a máxima dependência para realização das ABVD equivale a mínima pontuação ( 0 pontos) na escala, enquanto o valor máximo de 100 pontos equivale a independência total para as mesmas ABVD avaliadas ${ }^{11}$.

A Escala de Lawton avalia o nível de independência para realização de AIVD, ou seja, atividades necessárias para uma vida independente na comunidade, tais como: usar o telefone, fazer compras, utilizar transporte, administrar seu próprio dinheiro, preparar uma refeição, tomar seus remédios e executar atividades domésticas. Cada uma das questões tem três opções, sendo 0 = independência; 1 = dependência parcial; 2 = dependência total, onde a soma dos valores de cada item classifica o indivíduo em independente ou dependente, sendo que quanto maior o escore, maior será o grau de independência. Avaliação dos resultados do Índice de Lawton: para cada questão, a primeira resposta significa independência (3 pontos), a segunda dependência parcial ou capacidade com ajuda (2 pontos) e a terceira, dependência (1 ponto). A pontuação máxima é 27 pontos e a mínima 9 pontos ${ }^{12}$. 
O Inventário de Percepção de Suporte Familiar (IPSF) é utilizado para mensurar a percepção de suporte familiar dos indivíduos. É composto por 42 questões objetivas, que possuem como opção de resposta: quase nunca, às vezes, quase sempre. É dividido em três dimensões: Afetivo - Consistente, Adaptação Familiar e Autonomia. A classificação de pontos no domínio Afetividade-Consistência ocorre da seguinte forma: baixo (0-21 pontos), médio-baixo (22-28 pontos), médio-alto (29-33 pontos), e alto (34-42 pontos), sendo que quanto maior o escore obtido, pior é a percepção do suporte familiar. No domínio Adaptação-familiar, segundo fator, a pontuação ocorre de maneira invertida, ou seja, quanto maior a pontuação alcançada, melhor é o suporte familiar percebido, sendo a pontuação classificada em: baixo (0-18 pontos), médio-baixo (19-21 pontos), médio-alto (22-23 pontos), alto (24-26 pontos. No terceiro fator, domínio Autonomia, quanto maior a pontuação obtida pior é a percepção do suporte familiar, sendo que a pontuação obtida é classificada da seguinte maneira: baixo (0-9 pontos), médio-baixo (10-12 pontos), médio-alto (13-14 pontos), alto (15-16 pontos) ${ }^{13}$.

Os dados coletados foram tabulados em planilhas no Microssoft Excel e analisados por meio do Statistical Package for the Social Science (SPSS) versão 20.0, sendo realizada análise descritiva das variáveis de estudo.

Os procedimentos de coleta de informações foram instituídos após aprovação pelo Comitê de Ética da Universidade Estadual do Sudoeste da Bahia - UESB sob o parecer n 358.702 obedecendo à Resolução 466/12 que trata do Código de Ética da Pesquisa envolvendo Seres Humanos. Para participar da pesquisa voluntariamente o idoso ou seu responsável e o cuidador assinou um Termo de Consentimento Livre e Esclarecido.

\section{RESULTADO}

Constatou-se uma maior frequência de idosos do sexo feminino (59,0\%), na faixa etária de 80 a 84 anos (57,3\%) e não alfabetizados (51,3\%). Conforme dados da Tabela 1.

Tabela 1. Distribuição dos idosos longevos de acordo com as variáveis sociodemográficas. Jequié-BA, 2018.

\begin{tabular}{lll} 
& N & $\%$ \\
\hline Sexo & & \\
Masculino & 48 & 41 \\
Feminino & 69 & 59 \\
Faixa Etária & & 57,3 \\
80 a 84 & 67 & 42,7 \\
Acima de 84 & 50 & \\
Escolaridade & & 48,7 \\
Sim & 57 & 51,3 \\
Não & 60 & 100 \\
\hline Total & 117 & \\
\hline
\end{tabular}

Fonte: Dados da pesquisa. 
Na avaliação das ABVD, verificou- se que $59,0 \%$ dos idosos foram classificados como independentes. Entretanto nas atividades AIVD averiguou-se que 80,3\% foram classificados como dependentes.

Tabela 2. Distribuição dos idosos segundo as Atividades Básicas da Vida Diária (ABVD) e Atividades Instrumentais da Vida Diária (AIVD). Jequié-BA, 2018.

\begin{tabular}{lll} 
& $\mathbf{n}$ & \% \\
\hline ABVD & & \\
Dependente & 48 & 41 \\
Independente & 69 & 59 \\
Classificação da ABVD & & \\
Independente & 69 & 59 \\
Alta probabilidade de continuar a viver na comunidade & 47 & 40,2 \\
Importante dependência & 1 & 0,9 \\
AlVD' & & \\
Dependente & 94 & 80,3 \\
Independente & 23 & 19,7 \\
Classificação da AIVD & & \\
Independente & 23 & 19,7 \\
Dependência Parcial & 94 & 80,3 \\
\hline Total & 117 & 100 \\
\hline Fonte: Dados da pesquisa & &
\end{tabular}

${ }^{1}$ Atividades Básicas de Vida Diária, ${ }^{2}$ Atividades Instrumentais de Vida Diária

$\mathrm{Na}$ avaliação da percepção do suporte familiar, no domínio Afetividade-Consistência, observou-se uma maior predominância da pontuação alta $(62,4 \%)$. No domínio Adaptação-familiar, houve uma maior frequência de idosos com pontuação alta (53,0\%). Da mesma forma, no domínio Autonomia houve uma maior distribuição de idosos com pontuação alta $(60,7 \%)$.

Tabela 3. Distribuição da pontuação dos idosos longevos no Inventário de Percepção do Suporte Familiar (IPSF). JequiéBA, 2018.

\begin{tabular}{lll} 
& $\mathbf{N}$ & $\%$ \\
\hline Afetividade Consistência & & \\
Baixo (0-21 pontos) & 14 & 12,0 \\
Médio-baixo (22-28 pontos) & 8 & 6,8 \\
Médio-alto (29-33 pontos) & 22 & 18,8 \\
Alto (34-42 pontos) & 73 & 62,4
\end{tabular}




\begin{tabular}{lll} 
Adaptação familiar & & \\
Baixo (0-18 pontos) & 20 & 17,1 \\
Médio-baixo (19-21 pontos) & 15 & 12,8 \\
Médio-alto (22-23 pontos) & 20 & 17,1 \\
Alto (24-26 pontos) & 62 & 53,0 \\
Autonomia & & \\
Baixo (0-9 pontos) & 6 & 5,1 \\
Médio-baixo (10-12 pontos) & 13 & 11,1 \\
Médio-alto (13-14 pontos) & 27 & 23,1 \\
Alto (15-16 pontos) & 71 & 60,7 \\
\hline Total & 117 & 100,0 \\
\hline
\end{tabular}

Fonte: Dados da pesquisa

\section{DISCUSSÃO}

A amostra do presente estudo foi composta por 117 participantes, sendo que houve uma predominância do sexo feminino assim como em outros estudos realizados com idosos ${ }^{14-15}$. Essa feminização na terceira idade ocorre devido a proteção hormonal do estrogênio, a menor exposição a determinados fatores de risco, notadamente no trabalho, a menor prevalência do uso de álcool e drogas, levando a uma menor exposição a doenças cardiovasculares e câncer, e principalmente pela postura quanto a preocupação em relação a assistência à saúde ${ }^{16}$.

No que se refere a faixa etária a mais encontrada foi de 80 a 84 anos com predominância de sendo que outras pesquisas ${ }^{17-18}$ corroboram com os achados do presente estudo. Esses dados promovem a reflexão que os cuidadores e profissionais de saúde necessitam de capacitação para atender de maneira satisfatória os cuidados específicos e demandas dessa população que só tende a aumentar ${ }^{19}$.

No presente estudo, a maioria dos idosos mostrou-se com baixo nível de escolaridade, similar ao encontrado por outros autores $^{17-20}$. Provavelmente esses índices quanto a escolaridade sejam reflexo da baixa taxa de alfabetização do século passado, um tempo no qual não havia cobrança pela instrução escolar como atualmente ${ }^{19}$.

Com relação à capacidade para realizar as atividades básicas de vida diária, verificou-se uma maior frequência de idosos classificados como independentes. Em um estudo ${ }^{21}$ que teve como objetivo verificar a prevalência de incapacidade funcional e dependência de 100 idosos em um centro escola, os resultados encontrados corroboram com o presente estudo, onde se observou uma maior independência nos idosos segundo o Índice de Bhartel. Dados semelhantes foram encontrados em um estudo anterior ${ }^{20}$, que avaliou a capacidade de 116 idosos da comunidade para desenvolver ABVD e AIVD, encontrando que $57,9 \%$ dos idosos eram independentes para desempenhar tais atividades. 
A maior parte do dia dos idosos é preenchida com a realização de atividades necessárias, ou seja, atividades básicas, e isto pode sugerir que mesmo gastando maior tempo o idoso consegue executar a tarefa ${ }^{22}$. Ratificando, a baixa prevalência de dependência encontrada nas ABVD pode ser esclarecida pela natureza das tarefas realizadas, pois correspondem às funções mais elementares no que diz respeito à sobrevivência do indivíduo 5 .

Em um estudo que avaliou a influência dos fatores biopsicossociais sobre a capacidade funcional de idosos residente no nordeste do Brasil $^{5}$ foi encontrado uma prevalência de $52,6 \%$ de dependência para realização das AIVD. Segundo os autores esse maior índice de dependência ocorre pelo fato de as AIVD serem tarefas mais complexas, ou seja, ampliam os aspectos abordados pelas ABVD.

$\mathrm{Na}$ avaliação das AIVD verificou-se uma maior distribuição de idosos classificados como dependentes. Colaborando com nossos achados um estudo ${ }^{20}$ obteve uma dependência nas AIVD em 72,6\% da sua amostra. Essa dependência ocorre, pois, as AIVD demandam maior integridade física e cognitiva comparada às ABVD, ressaltando que a realização de uma tarefa abrange a interação de diversos sistemas fisiológicos, como o nervoso e o musculoesquelético que, na maioria das vezes encontra-se em decadência nos idosos octogenários ${ }^{23}$.

A incapacidade para realização de uma dessas AIVD, prejudica a vida social do idoso, e traz transtornos para o mesmo e sua família, a qual, dependendo da atividade, precisará de mais tempo disponível, recursos financeiros e energia para prover as demandas existentes ${ }^{20}$.

Segundo o Inventário de Percepção do Suporte Familiar, os idosos do presente estudo apresentaram comprometimento na percepção do suporte familiar em 2 domínios, Afetividade-Consistência e Autonomia, demonstrando dessa forma que os idosos possuem uma boa adaptação familiar, porém a convivência destes, não se apresenta adequada em relação a autonomia e a afetividade consistência, o que pode interferir na qualidade de vida desses idosos.

O domínio Afetividade-Consistência é definido como o vínculo emocional que os membros da família possuem uns com os outros, e envolve questões a respeito de limites, alianças, tempo desfrutado juntos, tomada de decisões, interesses e passatempos, bem como a habilidade nas estratégias de enfrentamento de situações-problema ${ }^{24}$.

A apresentação de escores elevados nesse domínio pela maioria dos idosos, evidencia que as famílias não estão preparadas para vivenciar emoções adequadas diante uma gama de estímulos, tanto de bem-estar quanto de mal-estar. Sendo assim, esse despreparo familiar ocasiona prejuízos na qualidade do cuidado prestado ao idoso ${ }^{13}$. 0 domínio Adaptação Familiar refere-se à expressão de bons sentimentos para com a família, como inclusão, compreensão, pertencimento, conversação e respeito ${ }^{25}$. Os dados encontrados nesse estudo, revelam que a maioria das famílias sabem expressar bons sentimentos para com os seus membros.

O domínio Autonomia Familiar, avalia as relações de confiança, privacidade e liberdade entre os membros da família24. Como resultado da pesquisa foi evidenciado que os idosos não possuem incentivo para se expressar e ter autonomia no ambiente familiar. Em um estudo para avaliar o suporte familiar em idosos residentes em domicílio ${ }^{25}$ foram 
encontrados dados semelhantes ao nossos, no qual, os idosos apresentaram uma maior frequência de percepção do suporte familiar inadequada nos domínios Afetividade-Consistência (52,0\%) e autonomia (58,0\%), sendo que no domínio Adaptação Familiar, houve uma maior classificação de idosos com percepção do suporte familiar adequado (67,3\%). Percebe-se que são poucos os estudos sobre ABVD, AIVD e Suporte Familiar em população longeva, entretanto, os resultados dos estudos realizados com idosos acima de 60 anos corroboram com os encontrados no presente estudo.

\section{CONSIDERAÇÕES FINAIS}

O perfil do grupo de idosos da área pesquisada, revelou uma população predominantemente feminina, na faixa etária de 80 a 84 anos e não alfabetizados. Em relação a capacidade funcional houve uma predominância de independência para realização das ABVD, enquanto para realização das AIVD foi observada uma dependência, indicando um estado inicial de declínio funcional. Quanto ao suporte familiar ficou evidenciado que os idosos do estudo possuem comprometimento nos domínios Afetividade-Consistência e Autonomia, o que demonstra em parte, que o suporte familiar não é oferecido da melhor maneira, sendo possível, a partir daí, orientar intervenções específicas para que a família ofereça ao idoso todo o suporte necessário para uma vida com boa qualidade.

\section{REFERÊNCIAS}

1. Sposito G, Neri AL, Yassuda MS. Cognitive performance and engagement in physical, social and intellectual activities in older adults: The FIBRA study. Dement neuropsychol [Internet]. 2015;9(3):270-78.

2. Pinto AH, Lange C, Albereci Pastore C, de Llano PM, Przylynski Castro D, dos Santos F. Capacidade funcional para atividades da vida diária de idosos da Estratégia de Saúde da Família da zona rural. Cienc Saude Colet 2016; 21(11): 3545-3555.

3. Barbosa BR, Almeida JM, Barbosa MR, Rossi-Barbosa LAR. Avaliação da capacidade funcional dos idosos e fatores associados à incapacidade. Cienc Saude Colet 2014;19(8): 3317-3325.

4. Figueiredo IM, Sampaio RF, Mancini MC, Silva FC, Souza MA. Teste de força de preensão utilizando o dinamômetro Jamar. Acta Fisiatr 2016; 14(2):104-10. 
5. Pavasini R, Guralnik J, Brown JC, di Bari M, Cesari M, Landi F, Vaes B, Legrand D, Verghese J, Wang C, Stenholm S, Ferrucci L, Lai JC, Bartes AA, Ferrer JEM, Lim Jae-Y, Ensrud KE, Cawthon P, Turusheva A, Frolova E, Rolland Y, Lauwers V, Corsonello A, Kirk GD, Ferrari R, Volpato S, Campo G. Short Physical Performance Battery and all-cause mortality: systematic review and meta-analysis. BMC Med 2016; 14: 215.

6. Busch TA, Duarte YA, Nunes DP, Lebrão ML, Naslavsky MS, Rodrigues AS, Amaro E. Factors associated with lower gait speed among the elderly living in a developing country: a cross-sectional population-based study. BMC Geriatr, 2015;15: 35-43.

7. Possatto JM, Rabelo DF. Ansiedade e depressão em idosos: associações com idade, sexo, capacidade funcional e suporte social. Rev Kairos 2017; 20(2):45-58.

8. Miranda GMD, Mendes AC, Silva ALA. O envelhecimento populacional brasileiro: desafios e consequências sociais atuais e futuras. Rev Bras Geriatr Gerontol 2016; 19(3): 507-519.

9. Berlezi EM, Farias AM, Dallazen F, Oliveira KR, Pillatt AP, Fortes CK. Como está a capacidade funcional de idosos residentes em comunidades com taxa de envelhecimento populacional acelerado? Rev Bras Geriatr Gerontol 2016; 19(4):643-652.

10. Bertolucci PH, Brucki SM, Campacci SR, Juliano Y. O mini-exame do estado mental em uma população geral: impacto da escolaridade. Arq Neuropsiquiatr. 1994;(52):1-7

11. Mahoney FI, Barthel DW. Functional evaluation: the Barthel Index. Maryland State Med J. 1965;(14):61-65.

12. Lawton MP, Brody EM. Assesment of older people: self-maintaining and instrumental activities of daily living. Gerontologist. 1969;(9):179-85

13. Wendt CJK, Aires M, Paz AA, Fengler FL, Paskulin LMG. Famílias de idosos na Estratégia de Saúde no Sul do Brasil. Rev Bras Enferm [Internet]. 2015;68(3):406-13. 
14. Nunes JD, Saes MO, Nunes BP, Siqueira FCV, Soares DC, Fassa MEG et al. Indicadores de incapacidade funcional e fatores associados em idosos: estudo de base populacional em Bagé, Rio Grande do Sul. Epidemiol. Serv. Saúde [Internet]. 2017; 26(2): 295-304.

15. Lopes MCBT, Lage JSS, Vancini-Campanharo CR, Okuno MFP, Batista REA. Fatores associados ao comprometimento funcional de idosos internados no serviço de emergência. Einstein (São Paulo). 2015;13(2):209-14.

16. Silva AG, Queirós A, Sa-Couto P, Rocha NP. Self-Reported Disability: Association With Lower Extremity Performance and Other Determinants in Older Adults Attending Primary Care. Phys Ther. 2015; 95(12):1628-37.

17. Medeiros KKAS, Junior EPP, Bousquat A, Medina MG O desafio da integralidade no cuidado ao idoso, no âmbito da Atenção Primária à Saúde. Saúde Debate. 2017; 41(3):288-295.

18. Veloso MV. et al. Functional Dependence in Institutionalized Elderly People and Memory Deficit. Revista ibero-americana de saúde e envelhecimento, 2016; 2(3).

19. Nogueira PSF, Marques MB, Coutinho JFV, Maia JC, Silva MJ, Moura ERF. Factors associated with the functional capacity of older adults with leprosy. Rev Bras Enferm 2017; 70(4):711-8.

20. Brito KQD, Menezes TN, Olinda RA. Functional disability and socioeconomic and demographic factors in elderly. Rev Bras Enferm 2015; 68(4):633-41.

21. McGrath RP, Ottenbacher KJ, Vincent BM, Kraemer WJ, Peterson MD. Muscle weakness and functional limitations in an ethnically diverse sample of older adults. Ethn Health 2017; 26:1-12.

22. Pereira LC, Prestes J, Melo GF, Neto LS, Funghetto SS, Pires AB, Boff G, Alves AT, de Oliveira Karnikowski MG. A influência da composição corporal na força de homens idosos brasileiros. Rev Bras Med Esporte, 2015; 21(3):196-9.

23. Chaves RN et al. Perda Cognitiva e Dependência Funcional em Idosos Longevos Residentes em Instituições de Longa Permanência. Revista cogitaire enfermagem, 2017; 22 (1). 
24. Margutti KMM, Schuch NJ, Schwanke CHA. Marcadores inflamatórios, sarcopenia e seus critérios diagnósticos em idosos: uma revisão sistemática. Rev Bras Geriatr Gerontol 2017; 20(3): 444-456.

25. Lopes GL, Santos MIPO. Funcionalidade de idosos cadastrados em uma unidade da Estratégia Saúde da Família segundo categorias da Classificação Internacional de Funcionalidade. Rev. bras. geriatr. gerontol, 2015;18(1): 71-83. 